\title{
MINIMIZATION PROBLEMS FOR CERTAIN STRUCTURED MATRICES *
}

\author{
ZHONGYUN LIU ${ }^{\dagger}$, RUI RALHA ${ }^{\ddagger}$, YULIN ZHANG ${ }^{\ddagger}$, AND CARLA FERREIRA $\ddagger$
}

\begin{abstract}
For given $Z, B \in \mathbb{C}^{n \times k}$, the problem of finding $A \in \mathbb{C}^{n \times n}$, in some prescribed class $\mathcal{W}$, that minimizes $\|A Z-B\|$ (Frobenius norm) has been considered by different authors for distinct classes $\mathcal{W}$. Here, we study this minimization problem for two other classes which include the symmetric Hamiltonian, symmetric skew-Hamiltonian, real orthogonal symplectic and unitary conjugate symplectic matrices. We also consider (as others have done for other classes $\mathcal{W}$ ) the problem of minimizing $\|A-\tilde{A}\|$ where $\tilde{A}$ is given and $A$ is a solution of the previous problem.

The key idea of our contribution is the reduction of each one of the above minimization problems to two independent subproblems in orthogonal subspaces of $\mathbb{C}^{n \times n}$. This is possible due to the special structures under consideration. We have developed MATLAB codes and present the numerical results of some tests.
\end{abstract}

Key words. Least-squares approximation, centralizer of $\mathcal{J}$, anticentralizer of $\mathcal{J}$, Moore-Penrose inverse

AMS subject classifications. 65F20, 65F 30

1. Introduction. Structured matrices appear in many applications and, in general, exploiting the structure may enable the development of more accurate algorithms. Such algorithms may also be more economic both in computation and storage and, in the application where such problems do occur, the structured solutions may have a more precise physical meaning. To mention just a few works related to this topic, the notion of strongly stable algorithms was introduced in [1] and used in the context of solving Toeplitz linear systems, eigensolvers for matrices of several special structures have been given in [2], a chart of backward errors for structured eigenvalue problems was presented in [10].

Skew-Hamiltonian matrices and Hamiltonian matrices are two important classes of structured matrices. In this paper we are interested in certain classes that include symmetric skewHamiltonian matrices and symmetric Hamiltonian matrices. Symmetric skew-Hamiltonian matrices arise in quantum mechanical problems with time reversal symmetry. Symmetric Hamiltonian matrices appear in response theory, more exactly in the study of closed shell Hartree-Fock wave functions, and also in solving algebraic Riccati equations in the context

*The research of the first author was supported by the National Natural Science Foundation of China, $\mathrm{N}^{\mathrm{o}}$. 11371075. The other authors were supported by the Research Centre of Mathematics of the University of Minho with the Portuguese Funds from the "Fundação para a Ciência e a Tecnologia", through the Project PEstOE/MAT/UI0013/2014.

${ }^{\dagger}$ School of Mathematics and Computing Science, Changsha University of Science \& Technology, Hunan, 410076, China (liuzhongyun@263.net).

${ }^{\ddagger}$ Mathematics Centre and Mathematics and Applications Department, University of Minho, 4710-057 Braga, Portugal (zhang@math.uminho.pt, caferrei@math.uminho.pt, r_ralha@math.uminho.pt). 
of continuous time linear quadratic optimal control problems. See [10] for references dealing with such applications.

We will be concerned with the following problems in certain structured classes $\mathcal{W}$ of matrices of size $n \times n$ (to be defined in the next section):

Problem I. Given $Z, B \in \mathbb{C}^{n \times k}$, find

$$
\sigma=\min _{A \in \mathcal{W}}\|A Z-B\|
$$

and characterize the class

$$
\mathcal{S}=\{A \in \mathcal{W}:\|A Z-B\|=\sigma\} .
$$

In particular, find necessary and sufficient conditions for the existence of $A \in \mathcal{W}$ such that $A Z=B$ and give a general form for $A$.

Problem II. Given $\widetilde{A} \in \mathbb{C}^{n \times n}$, find

$$
\widetilde{\sigma}=\min _{A \in \mathcal{S}}\|\widetilde{A}-A\|
$$

and find $A \in \mathcal{S}$ such that $\|\widetilde{A}-A\|=\widetilde{\sigma}$.

The above problems have been studied by many authors in various contexts and different classes of structured matrices were considered: centrosymmetric matrices [13], centrohermitian matrices [7], hermitian $R$-symmetric and hermitian $R$-skew symmetric matrices [11], matrices which satisfy $R A S=A$ and $R A S=-A$ for $R$ and $S$ nontrivial involutions, i.e. $R^{2}=S^{2}=I$ and $R, S \neq \pm I$ (Trench referred to these matrices as $(R, S)$ symmetric and $(R, S)$-skew symmetric [12]). Some applications that lead to the above minimization problems are also described in these references. In [6], Problem II has also been considered for $A$ in the set $\mathcal{W}$ of generalized $K$-centrohermitian matrices with prescribed spectra.

In section 2 we present the classes of structured matrices to be studied and prove a fundamental theorem. Some more theoretical results are given in section 3. The derivation of the explicit solutions for our minimization problems is presented in section 4 . In section 5 we exhibit our structure-exploiting algorithms for computing those solutions. In section 6 we present results of our numerical experiments and finish with some conclusions in section 7 .

2. The centralizer and anticentralizer of $\mathcal{J}$. Let $\mathcal{J}=\left[\begin{array}{cc}0 & I \\ -I & 0\end{array}\right]$, where $I$ is the identity matrix of order $m$. In [2], the sets

$$
\mathscr{C}=\left\{A \in \mathbb{C}^{2 m \times 2 m} \mid A \mathcal{J}=\mathcal{J} A\right\}=\left\{\left[\begin{array}{cc}
D & -E \\
E & D
\end{array}\right] \mid D, E \in \mathbb{C}^{m \times m}\right\}
$$


and

$$
\mathscr{C}^{(a)}=\left\{A \in \mathbb{C}^{2 m \times 2 m} \mid A \mathcal{J}=-\mathcal{J} A\right\}=\left\{\left[\begin{array}{cc}
G & F \\
F & -G
\end{array}\right] \mid F, G \in \mathbb{C}^{m \times m}\right\}
$$

are called the centralizer of $\mathcal{J}$ and the anticentralizer of $\mathcal{J}$, respectively. Also it was observed that orthogonal sympletic matrices and unitary conjugate sympletic matrices belong to $\mathscr{C}$. It can be seen that symmetric skew-Hamiltonian matrices also belong to $\mathscr{C}$ and symmetric Hamiltonian matrices belong to $\mathscr{C}^{(a)}$.

The next theorem shows the relation between spaces $\mathbb{C}^{2 m \times 2 m}, \mathscr{C}$ and $\mathscr{C}^{(a)}$. We use the Frobenius inner product, $\langle X, Y\rangle=\operatorname{trace}\left(Y^{H} X\right)$ for matrices $X$ and $Y$. An orthogonal direct sum will be denoted with the symbol $\oplus^{\perp}$.

LEMMA 2.1. We have

$$
\mathbb{C}^{2 m \times 2 m}=\mathscr{C} \oplus^{\perp} \mathscr{C}^{(a)} .
$$

Proof. First, we show that every matrix in $\mathbb{C}^{2 m \times 2 m}$ can be written as a sum of two matrices, one in $\mathscr{C}$ and the other one in $\mathscr{C}^{(a)}$. Given $A \in \mathbb{C}^{2 m \times 2 m}$, let

$$
A_{c}=\frac{A-\mathcal{J} A \mathcal{J}}{2} \quad \text { and } \quad A_{s}=\frac{A+\mathcal{J} A \mathcal{J}}{2} .
$$

We have $A=A_{c}+A_{s}$ and

$$
\begin{aligned}
& \mathcal{J} A_{c} \mathcal{J}=\frac{\mathcal{J} A \mathcal{J}-\mathcal{J}^{2} A \mathcal{J}^{2}}{2}=\frac{\mathcal{J} A \mathcal{J}-A}{2}=-A_{c}, \\
& \mathcal{J} A_{s} \mathcal{J}=\frac{\mathcal{J} A \mathcal{J}+\mathcal{J}^{2} A \mathcal{J}^{2}}{2}=\frac{\mathcal{J} A \mathcal{J}+A}{2}=A_{s}
\end{aligned}
$$

that is, $A_{c} \in \mathscr{C}$ and $A_{s} \in \mathscr{C}^{(a)}$. This decomposition is unique since $\mathscr{C} \bigcap \mathscr{C}^{(a)}=\{O\}$. In fact, if $A \in \mathscr{C} \cap \mathscr{C}^{(a)}$, then $\mathcal{J} A \mathcal{J}=-A$ and $\mathcal{J} A \mathcal{J}=A$, which implies that $A=O$. So, we have a direct sum.

Finally, let us see that $\mathscr{C} \perp \mathscr{C}^{(a)}$. If $X \in \mathscr{C}$ and $Y \in \mathscr{C}^{(a)}$, we have $\mathcal{J} X \mathcal{J}=-X$ and $\mathcal{J} Y \mathcal{J}=Y$. Since $\mathcal{J}^{H} \mathcal{J}=\mathcal{J} \mathcal{J}^{H}=I$, we have

$$
\begin{aligned}
\langle X, Y\rangle & =\langle-\mathcal{J} X \mathcal{J}, \mathcal{J} Y \mathcal{J}\rangle \\
& =-\operatorname{trace}\left[(\mathcal{J} Y \mathcal{J})^{H} \mathcal{J} X \mathcal{J}\right]=-\operatorname{trace}\left(\mathcal{J}^{H} Y^{H} X \mathcal{J}\right) \\
& =-\operatorname{trace}\left(Y^{H} X\right)=-\langle X, Y\rangle
\end{aligned}
$$

which means that $\langle X, Y\rangle=0$ and, thus, $\mathscr{C} \perp \mathscr{C}^{(a)}$. $\square$

The minimization problems that we are tackling here have not been considered in [2]. As compared to the work in [12], for $(R, S)$ symmetric and $(R, S)$-skew symmetric matrices, we 
emphasize that matrices in $\mathscr{C}$ and $\mathscr{C}^{(a)}$ do not fall into these classes as $\mathcal{J}$ is not an involution $\left(\mathcal{J}^{2} \neq I\right)$. The key idea of our contribution is the reduction of each one of the minimization problems under consideration to two independent subproblems in orthogonal subspaces of $\mathbb{C}^{2 m \times 2 m}$.

3. Preliminary results. The following result has been given in [2, Lemma 2.5].

Lemma 3.1. Let $P \in \mathbb{C}^{2 m \times 2 m}$ be the unitary matrix given by

$$
P=\frac{\sqrt{2}}{2}\left[\begin{array}{cc}
I & I \\
i I & -i I
\end{array}\right]
$$

where $I$ is the identity matrix of order $m$. We have:

(i) if $A \in \mathscr{C}$ (as defined in (2.1)), then

$$
P^{H} A P=\left[\begin{array}{ll}
M_{1} & \\
& N_{1}
\end{array}\right],
$$

where $M_{1}=D-i E$ and $N_{1}=D+i E$;

(ii) if $A \in \mathscr{C}^{(a)}$ (as defined in (2.2)), then

$$
P^{H} A P=\left[\begin{array}{ll} 
& M_{2} \\
N_{2} &
\end{array}\right],
$$

where $M_{2}=G-i F$ and $N_{2}=G+i F$.

Using Lemma 3.1 and Lemma 2.1, for any matrix $\tilde{A} \in \mathbb{C}^{2 m \times 2 m}$ we have

$$
P^{H} \tilde{A} P=P^{H}\left(A_{c}+A_{s}\right) P=\left[\begin{array}{ll}
M_{1} & \\
& N_{1}
\end{array}\right]+\left[\begin{array}{ll} 
& M_{2} \\
N_{2} &
\end{array}\right],
$$

where $A_{c}$ and $A_{s}$ are as defined in (2.3). For the sake of convenience, we will use $M$ to represent either $M_{1}$ or $M_{2}$ if there is no danger of ambiguity. The same applies to $N, N_{1}$ and $N_{2}$.

To tackle Problem I, we now define two new subspaces

$$
\mathcal{C}_{\mathcal{J}}^{2 m \times k}=\left\{X \in \mathbb{C}^{2 m \times k}: i \mathcal{J} X=X\right\}=\left\{\left[\begin{array}{c}
\widehat{X} \\
-i \widehat{X}
\end{array}\right] \mid \widehat{X} \in \mathbb{C}^{m \times k}\right\}
$$

and

$$
\mathcal{S}_{\mathcal{J}}^{2 m \times k}=\left\{Y \in \mathbb{C}^{2 m \times k}: i \mathcal{J} Y=-Y\right\}=\left\{\left[\begin{array}{c}
\widehat{Y} \\
i \widehat{Y}
\end{array}\right] \mid \widehat{Y} \in \mathbb{C}^{m \times k}\right\} .
$$

Any matrix $B \in \mathbb{C}^{2 m \times k}$ can be expressed as the sum of one matrix in $\mathcal{C}_{\mathcal{J}}^{2 m \times k}$ and another in $\mathcal{S}_{\mathcal{J}}^{2 m \times k}$. 
Lemma 3.2. We have

$$
\mathbb{C}^{2 m \times k}=\mathcal{C}_{\mathcal{J}}^{2 m \times k} \oplus^{\perp} \mathcal{S}_{\mathcal{J}}^{2 m \times k} .
$$

In fact, any matrix $B \in \mathbb{C}^{2 m \times k}$ can be written as

$$
B=\frac{B+i \mathcal{J} B}{2}+\frac{B-i \mathcal{J} B}{2},
$$

where the first matrix in the right hand side belongs to $\mathcal{C}_{\mathcal{J}}^{2 m \times k}$ and the second to $\mathcal{S}_{\mathcal{J}}^{2 m \times k}$. The rest of the proof is similar to the one given for the previous result. This theorem plays a major role in Problem I. Furthermore, we have the following lemma.

Lemma 3.3. Let $P$ be the unitary matrix defined in (3.1). If $X \in \mathcal{C}_{\mathcal{J}}^{2 m \times k}$ and $Y \in \mathcal{S}_{\mathcal{J}}^{2 m \times k}$, then

$$
P^{H} X=\sqrt{2}\left[\begin{array}{c}
O \\
\widehat{X}
\end{array}\right] \quad \text { and } \quad P^{H} Y=\sqrt{2}\left[\begin{array}{c}
\widehat{Y} \\
O
\end{array}\right]
$$

The proof is straightforward if we perform the matrix products. It is also straightforward to prove the following result concerning the invariance (and swapping) of the subspaces $\mathcal{C}_{\mathcal{J}}^{2 m \times k}$ and $\mathcal{S}_{\mathcal{J}}^{2 m \times k}$ under $A \in \mathscr{C}$ (and $A \in \mathscr{C}^{(a)}$ ).

Lemma 3.4. Let $X \in \mathcal{C}_{\mathcal{J}}^{2 m \times k}$ and $Y \in \mathcal{S}_{\mathcal{J}}^{2 m \times k}$.

1. For $A \in \mathscr{C}$, we have $A X \in \mathcal{C}_{\mathcal{J}}^{2 m \times k}$ and $A Y \in \mathcal{S}_{\mathcal{J}}^{2 m \times k}$.

2. For $A \in \mathscr{C}^{(a)}$, we have $A X \in \mathcal{S}_{\mathcal{J}}^{2 m \times k}$ and $A Y \in \mathcal{C}_{\mathcal{J}}^{2 m \times k}$.

4. The minimization problems. In this section we recall two results concerning the minimization problem for the general case, that is, given matrices $Z \in \mathbb{C}^{q \times k}$ and $B \in \mathbb{C}^{p \times k}$, we seek $\min _{A \in \mathbb{C}^{p \times q}}\|A Z-B\|$, when there is no constraint on $A$. We also give a different interpretation of the known necessary and sufficient condition for such minimum to be zero. Then we turn to the problem with $A \in \mathscr{C}$ and $A \in \mathscr{C}^{(a)}$, exploiting the structure properties of these matrices.

4.1. General case. For a matrix $Z \in \mathbb{C}^{q \times k}$, we will use the standard notation $Z^{\dagger}$ for the Moore-Penrose inverse. We recall that throughout the paper we use the Frobenius norm, the norm induced by the Frobenius inner product.

Lemma 4.1. [12, Lemma 2] If $Z \in \mathbb{C}^{q \times k}$ and $B \in \mathbb{C}^{p \times k}$, then

$$
\min _{A \in \mathbb{C}^{p \times q}}\|A Z-B\|=\left\|B\left(I-Z^{\dagger} Z\right)\right\|,
$$

and this minimum is attained if and only if

$$
A=B Z^{\dagger}+K\left(I-Z Z^{\dagger}\right)
$$


with $K \in \mathbb{C}^{p \times q}$ arbitrary. Moreover, $A_{0}=B Z^{\dagger}$ is the unique matrix of this form with minimum norm.

In particular, we have $A Z=B$ if and only if $B\left(I-Z^{\dagger} Z\right)=O([12$, Lemma 3]). For $q \geq k$, if $Z$ is full $\operatorname{rank}^{1}$ then $Z^{\dagger} Z=I$ and the previous condition holds. More generally, we have the following result.

Theorem 4.2. Given $Z$ in $\mathbb{C}^{q \times k}$, with $q \geq k$, and $B$ in $\mathbb{C}^{p \times k}$, we have $B\left(I-Z^{\dagger} Z\right)=O$ if and only if the null space of $Z$ is contained in the null space of $B$, that is, $\operatorname{null}(Z) \subseteq \operatorname{null}(B)$.

Proof. To conclude that the given condition is necessary, note that from $B\left(I-Z^{\dagger} Z\right)=O$ we get that $Z x=0$ implies $B x=0$. Conversely, assume that $\operatorname{null}(Z) \subseteq \operatorname{null}(B)$. Then, $\operatorname{row}(Z)$ and $\operatorname{row}(B)$ are the orthogonal complements of $\operatorname{null}(Z)$ and $\operatorname{null}(B)$, respectively, and satisfy $\operatorname{row}(B) \subseteq \operatorname{row}(Z)$ [5, Theorems 3.12 and 3.11]. Thus, there is a matrix $M$ such that $B=M Z$ and, since $Z Z^{\dagger} Z=Z$,

$$
B\left(I-Z^{\dagger} Z\right)=M Z-M Z Z^{\dagger} Z=M Z-M Z=O .
$$

In the proofs of the results concerning Problem II we will use the following

Lemma 4.3. [12, Lemma 4] Suppose that $L \in \mathbb{C}^{p \times q}$ and $\Gamma \in \mathbb{C}^{q \times q}$ where $\Gamma^{2}=\Gamma=\Gamma^{H}$. Then the unique matrix of the form $L-M \Gamma$ with minimum Frobenius norm is $L-L \Gamma=L(I-\Gamma)$.

4.2. $A \in \mathscr{C}$ (centralizer of $\mathcal{J})$. We first consider Problem I.

Let $Z, B \in \mathbb{C}^{2 m \times k}(m \geq k)$. Write $Z=Z_{1}+Z_{2}$ with

$$
Z_{1}=\frac{Z+i \mathcal{J} Z}{2}, \quad Z_{2}=\frac{Z-i \mathcal{J} Z}{2},
$$

and $B=B_{1}+B_{2}$ with

$$
B_{1}=\frac{B+i \mathcal{J} B}{2}, \quad B_{2}=\frac{B-i \mathcal{J} B}{2},
$$

where $Z_{1}, B_{1} \in \mathcal{C}_{\mathcal{J}}^{2 m \times k}$ and $Z_{2}, B_{2} \in \mathcal{S}_{\mathcal{J}}^{2 m \times k}$.

From Lemma 3.4, for $A \in \mathscr{C},\left(A Z_{1}-B_{1}\right) \in \mathcal{C}_{\mathcal{J}}^{2 m \times k}$ and $\left(A Z_{2}-B_{2}\right) \in \mathcal{S}_{\mathcal{J}}^{2 m \times k}$. The orthogonality of these subspaces implies

$$
\|A Z-B\|^{2}=\left\|\left(A Z_{1}-B_{1}\right)+\left(A Z_{2}-B_{2}\right)\right\|^{2}=\left\|A Z_{1}-B_{1}\right\|^{2}+\left\|A Z_{2}-B_{2}\right\|^{2} .
$$

\footnotetext{
${ }^{1}$ Since $q \geq k$, by full rank we mean full column rank.
} 
According to (3.4) and (3.5),

$$
Z_{1}=\left[\begin{array}{c}
\widehat{Z}_{1} \\
-i \widehat{Z}_{1}
\end{array}\right], \quad Z_{2}=\left[\begin{array}{c}
\widehat{Z}_{2} \\
i \widehat{Z}_{2}
\end{array}\right], \quad B_{1}=\left[\begin{array}{c}
\widehat{B}_{1} \\
-i \widehat{B}_{1}
\end{array}\right], \quad B_{2}=\left[\begin{array}{c}
\widehat{B}_{2} \\
i \widehat{B}_{2}
\end{array}\right]
$$

where $\widehat{Z}_{1}, \widehat{Z}_{2}, \widehat{B}_{1}, \widehat{B}_{2} \in \mathbb{C}^{m \times k}$.

For $A \in \mathscr{C}$, the invariance of the Frobenius norm under unitary multiplication, (3.2) and (3.6) imply that

$$
\begin{aligned}
\left\|A Z_{1}-B_{1}\right\|^{2}=\left\|P^{H} A P P^{H} Z_{1}-P^{H} B_{1}\right\|^{2} & =2\left\|\left[\begin{array}{ll}
M & \\
& N
\end{array}\right]\left[\begin{array}{c}
O \\
\widehat{Z}_{1}
\end{array}\right]-\left[\begin{array}{c}
O \\
\widehat{B}_{1}
\end{array}\right]\right\|^{2} \\
& =2\left\|N \widehat{Z}_{1}-\widehat{B}_{1}\right\|^{2} .
\end{aligned}
$$

Similarly,

$$
\left\|A Z_{2}-B_{2}\right\|^{2}=2\left\|M \widehat{Z}_{2}-\widehat{B}_{2}\right\|^{2}
$$

Therefore, our problem is reduced to two independent subproblems of the same type in the orthogonal subspaces, one for the pair $\left(Z_{1}, B_{1}\right)$ and the other for the pair $\left(Z_{2}, B_{2}\right)$. Thus, with respect to Problem I, we have the following result.

TheOREM 4.4. Let $Z, B \in \mathbb{C}^{2 m \times k}$. Consider $P$ as given in (3.1) and $\widehat{Z}_{1}, \widehat{Z}_{2}, \widehat{B}_{1}, \widehat{B}_{2}$ in (4.5). Then

$$
\min _{A \in \mathscr{C}}\|A Z-B\|=\sqrt{2}\left(\left\|\widehat{B}_{1}\left(I-\widehat{Z}_{1}^{\dagger} \widehat{Z}_{1}\right)\right\|^{2}+\left\|\widehat{B}_{2}\left(I-\widehat{Z}_{2}^{\dagger} \widehat{Z}_{2}\right)\right\|^{2}\right)^{1 / 2}
$$

and this minimum is attained if and only if

$$
A=P\left[\begin{array}{ll}
M & \\
& N
\end{array}\right] P^{H}=\frac{1}{2}\left[\begin{array}{cc}
M+N & -(M-N) i \\
(M-N) i & M+N
\end{array}\right]
$$

where $M=\widehat{B}_{2} \widehat{Z}_{2}^{\dagger}+K_{2}\left(I-\widehat{Z}_{2} \widehat{Z}_{2}^{\dagger}\right)$ and $N=\widehat{B}_{1} \widehat{Z}_{1}^{\dagger}+K_{1}\left(I-\widehat{Z}_{1} \widehat{Z}_{1}^{\dagger}\right)$ with arbitrary $K_{1}, K_{2} \in \mathbb{C}^{m \times m}$. Moreover, $A=P\left[\begin{array}{ll}\widehat{B}_{2} \widehat{Z}_{2}^{\dagger} & \\ & \widehat{B}_{1} \widehat{Z}_{1}^{\dagger}\end{array}\right] P^{H}$ is the unique matrix of this form with minimum norm.

The relations (4.6) and (4.7) follow from the application of Lemma 4.1 to $\left\|N \widehat{Z}_{1}-\widehat{B}_{1}\right\|$ and $\left\|M \widehat{Z}_{2}-\widehat{B}_{2}\right\|$.

Now we consider Problem II. 
Let $\sigma_{c}=\min _{A \in \mathscr{C}}\|A Z-B\| \quad$ and $\quad \mathcal{S}_{c}=\left\{A \in \mathscr{C}:\|A Z-B\|=\sigma_{c}\right\}$.

For $\widetilde{A} \in \mathbb{C}^{2 m \times 2 m}$, using Lemma 2.1 , there exists a unique pair of matrices $\widetilde{A}_{1} \in \mathscr{C}$, $\widetilde{A}_{2} \in \mathscr{C}^{(a)}$ such that $\widetilde{A}=\widetilde{A}_{1}+\widetilde{A}_{2}$ with

$$
\widetilde{A}_{1}=\frac{\widetilde{A}-\mathcal{J} \widetilde{A} \mathcal{J}}{2} \quad \text { and } \quad \widetilde{A}_{2}=\frac{\widetilde{A}+\mathcal{J} \widetilde{A} \mathcal{J}}{2} .
$$

Using Lemma 3.1, we write

$$
P^{H} \widetilde{A}_{1} P=\left[\begin{array}{ll}
M_{1} & \\
& N_{1}
\end{array}\right] \quad \text { and } \quad P^{H} \widetilde{A}_{2} P=\left[\begin{array}{ll} 
& M_{2} \\
N_{2} &
\end{array}\right] .
$$

THEOREM 4.5. Assume the conditions of Theorem 4.4. Let $\tilde{A} \in \mathbb{C}^{2 m \times 2 m}$ and $M_{1}, N_{1}, M_{2}, N_{2}$ given in (4.9). Then

$$
\min _{A \in \mathcal{S}_{c}}\|\widetilde{A}-A\|=\left(\left\|M_{2}\right\|^{2}+\left\|N_{2}\right\|^{2}+\left\|\left(M_{1} \widehat{Z}_{2}-\widehat{B}_{2}\right) \widehat{Z}_{2}^{\dagger}\right\|^{2}+\left\|\left(N_{1} \widehat{Z}_{1}-\widehat{B}_{1}\right) \widehat{Z}_{1}^{\dagger}\right\|^{2}\right)^{1 / 2}
$$

and this minimum is attained if and only if

$$
A=\frac{1}{2}\left[\begin{array}{cc}
M_{3}+N_{3} & -\left(M_{3}-N_{3}\right) i \\
\left(M_{3}-N_{3}\right) i & M_{3}+N_{3}
\end{array}\right],
$$

where $M_{3}=\widehat{B}_{2} \widehat{Z}_{2}^{\dagger}+M_{1}\left(I-\widehat{Z}_{2} \widehat{Z}_{2}^{\dagger}\right)$ and $N_{3}=\widehat{B}_{1} \widehat{Z}_{1}^{\dagger}+N_{1}\left(I-\widehat{Z}_{1} \widehat{Z}_{1}^{\dagger}\right)$.

Proof. Let $\widetilde{A}=\widetilde{A}_{1}+\widetilde{A}_{2}$, with $\widetilde{A}_{1}, \widetilde{A}_{2}$ given in (4.8), and $A \in \mathcal{S}_{c}$ given by (4.7). Since the Frobenius norm is invariant under unitary transformations, we have

$$
\begin{aligned}
\|\widetilde{A}-A\|^{2}= & \left\|\left(\tilde{A}_{1}-A\right)+\tilde{A}_{2}\right\|^{2} \\
= & \left\|P^{H}\left(\widetilde{A}_{1}-A\right) P+P^{H} \widetilde{A}_{2} P\right\|^{2} \\
= & \left\|\left[\begin{array}{cc}
M_{1}-\widehat{B}_{2} \widehat{Z}_{2}^{\dagger}-K_{2}\left(I-\widehat{Z}_{2} \widehat{Z}_{2}^{\dagger}\right) & N_{1}-\widehat{B}_{1} \widehat{Z}_{1}^{\dagger}-K_{1}\left(I-\widehat{Z}_{1} \widehat{Z}_{1}^{\dagger}\right)
\end{array}\right]\right\|^{2} \\
& =\left\|M_{2}\right\|^{2}+\left\|N_{2}\right\|^{2}+\left\|M_{1}-\widehat{B}_{2} \widehat{Z}_{2}^{\dagger}-K_{2}\left(I-\widehat{Z}_{2} \widehat{Z}_{2}^{\dagger}\right)\right\|^{2}+ \\
& \quad+\left\|N_{1}-\widehat{B}_{1} \widehat{Z}_{1}^{\dagger}-K_{1}\left(I-\widehat{Z}_{1} \widehat{Z}_{1}^{\dagger}\right)\right\|^{2} .
\end{aligned}
$$

Observe that only $K_{1}, K_{2} \in \mathbb{C}^{m \times m}$ are free. Thus,

$$
\begin{aligned}
\min _{A \in \mathcal{S}_{c}}\|\tilde{A}-A\|^{2}=\left\|M_{2}\right\|^{2} & +\left\|N_{2}\right\|^{2}+\min _{K_{2} \in \mathbb{C}^{m \times m}}\left\|M_{1}-\widehat{B}_{2} \widehat{Z}_{2}^{\dagger}-K_{2}\left(I-\widehat{Z}_{2} \widehat{Z}_{2}^{\dagger}\right)\right\|^{2}+ \\
& +\min _{K_{1} \in \mathbb{C}^{m \times m}}\left\|N_{1}-\widehat{B}_{1} \widehat{Z}_{1}^{\dagger}-K_{1}\left(I-\widehat{Z}_{1} \widehat{Z}_{1}^{\dagger}\right)\right\|^{2} .
\end{aligned}
$$


For $X \in \mathbb{C}^{m \times k}$, the properties of the Moore-Penrose inverse imply that $\left(I-X X^{\dagger}\right)^{2}=$ $\left(I-X X^{\dagger}\right)=\left(I-X X^{\dagger}\right)^{H}$. Thus, we can apply Lemma 4.3 to each of the two minimization problems on the right side of the previous equation. The minimum values are attained if and only if

$$
K_{2}=M_{1}-\widehat{B}_{2} \widehat{Z}_{2}^{\dagger} \quad \text { and } \quad K_{1}=N_{1}-\widehat{B}_{1} \widehat{Z}_{1}^{\dagger}
$$

which give

$$
\begin{aligned}
K_{2}\left(I-\widehat{Z}_{2} \widehat{Z}_{2}^{\dagger}\right) & =\left(M_{1}-\widehat{B}_{2} \widehat{Z}_{2}^{\dagger}\right)\left(I-\widehat{Z}_{2} \widehat{Z}_{2}^{\dagger}\right)=M_{1}\left(I-\widehat{Z}_{2} \widehat{Z}_{2}^{\dagger}\right)-\widehat{B}_{2} \widehat{Z}_{2}^{\dagger}+\widehat{B}_{2} \widehat{Z}_{2}^{\dagger} \widehat{Z}_{2} \widehat{Z}_{2}^{\dagger} \\
& =M_{1}\left(I-\widehat{Z}_{2} \widehat{Z}_{2}^{\dagger}\right)
\end{aligned}
$$

and

$$
K_{1}\left(I-\widehat{Z}_{1} \widehat{Z}_{1}^{\dagger}\right)=\left(N_{1}-\widehat{B}_{1} \widehat{Z}_{1}^{\dagger}\right)\left(I-\widehat{Z}_{1} \widehat{Z}_{1}^{\dagger}\right)=N_{1}\left(I-\widehat{Z}_{1} \widehat{Z}_{1}^{\dagger}\right) .
$$

Hence, the minimum values are

$$
\left\|M_{1}-\widehat{B}_{2} \widehat{Z}_{2}^{\dagger}-M_{1}\left(I-\widehat{Z}_{2} \widehat{Z}_{2}^{\dagger}\right)\right\|^{2}=\left\|\left(M_{1} \widehat{Z}_{2}-\widehat{B}_{2}\right) \widehat{Z}_{2}^{\dagger}\right\|^{2}
$$

and

$$
\left\|N_{1}-\widehat{B}_{1} \widehat{Z}_{1}^{\dagger}-N_{1}\left(I-\widehat{Z}_{1} \widehat{Z}_{1}^{\dagger}\right)\right\|^{2}=\left\|\left(N_{1} \widehat{Z}_{1}-\widehat{B}_{1}\right) \widehat{Z}_{1}^{\dagger}\right\|^{2} .
$$

This shows that $\min _{A \in \mathcal{S}_{c}}\|\widetilde{A}-A\|$ is given by (4.10) and it is attained if and only if $A$ takes the form (4.11).

4.3. $A \in \mathscr{C}^{(a)}$ (anticentralizer of $\mathcal{J}$ ). For $A \in \mathscr{C}^{(a)}$ the results are entirely analogous to the ones just presented for $A \in \mathscr{C}$ and therefore we will omit the proofs. In view of the fact that for $A \in \mathscr{C}^{(a)},\left(A Z_{2}-B_{1}\right) \in \mathcal{C}_{\mathcal{J}}^{2 m \times k}$ and $\left(A Z_{1}-B_{2}\right) \in \mathcal{S}_{\mathcal{J}}^{2 m \times k}$ are orthogonal, we may use arguments similar to those used in the previous subsection to get the following results.

TheOrem 4.6. Let $Z, B \in \mathbb{C}^{2 m \times k}$. Consider $P$ as given in (3.1) and $\widehat{Z}_{1}, \widehat{Z}_{2}, \widehat{B}_{1}, \widehat{B}_{2}$ in (4.5). Then

$$
\min _{A \in \mathscr{C}(a)}\|A Z-B\|=\sqrt{2}\left(\left\|\widehat{B}_{2}\left(I-\widehat{Z}_{1}^{\dagger} \widehat{Z}_{1}\right)\right\|^{2}+\left\|\widehat{B}_{1}\left(I-\widehat{Z}_{2}^{\dagger} \widehat{Z}_{2}\right)\right\|^{2}\right)^{1 / 2}
$$

and this minimum is attained if and only if

$$
A=P\left[\begin{array}{ll} 
& M \\
N &
\end{array}\right] P^{H}=\frac{1}{2}\left[\begin{array}{cc}
M+N & (M-N) i \\
(M-N) i & -(M+N)
\end{array}\right],
$$

where $M=\widehat{B}_{2} \widehat{Z}_{1}^{\dagger}+K_{2}\left(I-\widehat{Z}_{1} \widehat{Z}_{1}^{\dagger}\right)$ and $N=\widehat{B}_{1} \widehat{Z}_{2}^{\dagger}+K_{1}\left(I-\widehat{Z}_{2} \widehat{Z}_{2}^{\dagger}\right)$ with arbitrary $K_{1}, K_{2} \in \mathbb{C}^{m \times m}$. Moreover, $A=P\left[\widehat{B}_{1} \widehat{Z}_{2}^{\dagger}{ }^{\widehat{B}_{2}} \widehat{Z}_{1}^{\dagger}\right] P^{H}$ is the unique matrix of this form with minimum norm. 
Let $\sigma_{s}=\min _{A \in \mathscr{C}(a)}\|A Z-B\| \quad$ and $\quad \mathcal{S}_{s}=\left\{A \in \mathscr{C}^{(a)}:\|A Z-B\|=\sigma_{s}\right\}$.

THeOREM 4.7. Assume the conditions of Theorem 4.6. Let $\tilde{A} \in \mathbb{C}^{2 m \times 2 m}$ and $M_{1}, N_{1}, M_{2}, N_{2}$ given in (4.9). Then

$$
\min _{A \in \mathcal{S}_{s}}\|\tilde{A}-A\|=\left(\left\|M_{1}\right\|^{2}+\left\|N_{1}\right\|^{2}+\left\|\left(M_{2} \widehat{Z}_{1}-\widehat{B}_{2}\right) \widehat{Z}_{1}^{\dagger}\right\|^{2}+\left\|\left(N_{2} \widehat{Z}_{2}-\widehat{B}_{1}\right) \widehat{Z}_{2}^{\dagger}\right\|^{2}\right)^{1 / 2}
$$

and this minimum is attained if and only if

$$
A=\frac{1}{2}\left[\begin{array}{cc}
M_{4}+N_{4} & \left(M_{4}-N_{4}\right) i \\
\left(M_{4}-N_{4}\right) i & -\left(M_{4}+N_{4}\right)
\end{array}\right]
$$

where $M_{4}=\widehat{B}_{2} \widehat{Z}_{1}^{\dagger}+M_{2}\left(I-\widehat{Z}_{1} \widehat{Z}_{1}^{\dagger}\right)$ and $N_{4}=\widehat{B}_{1} \widehat{Z}_{2}^{\dagger}+N_{2}\left(I-\widehat{Z}_{2} \widehat{Z}_{2}^{\dagger}\right)$.

4.4. Further results on residuals. The conditions for the existence of solutions $A \in \mathscr{C}$ and $A \in \mathscr{C}^{(a)}$ such that $\|A Z-B\|=0$ are clear from (4.6) and (4.12), respectively. So, for $Z=Z_{1}+Z_{2}$ with $Z_{1} \in \mathcal{C}_{\mathcal{J}}^{2 m \times k}$ and $Z_{2} \in \mathcal{S}_{\mathcal{J}}^{2 m \times k}$, if $Z_{1}$ and $Z_{2}$ have full rank, a null residual is achieved with matrices given by (4.7) and (4.13). Now, it also follows from Theorem 4.2 that consistency will be achieved for $A \in \mathscr{C}$ even when $Z_{1}$ and/or $Z_{2}$ are not full rank if the null spaces of $\widehat{Z}_{1}$ and $\widehat{Z}_{2}$ are contained in the null spaces of $\widehat{B}_{1}$ and $\widehat{B}_{2}$, respectively. For $A \in \mathscr{C}^{(a)}$, the conditions are $\operatorname{null}\left(\widehat{Z}_{1}\right) \subseteq \operatorname{null}\left(\widehat{B}_{2}\right)$ and $\operatorname{null}\left(\widehat{Z}_{2}\right) \subseteq \operatorname{null}\left(\widehat{B}_{1}\right)$. Observing that $\operatorname{null}\left(\widehat{Z}_{1}\right)=\operatorname{null}\left(Z_{1}\right), \operatorname{null}\left(\widehat{Z}_{2}\right)=\operatorname{null}\left(Z_{2}\right), \operatorname{null}\left(\widehat{B}_{1}\right)=\operatorname{null}\left(B_{1}\right)$ and $\operatorname{null}\left(\widehat{B}_{2}\right)=\operatorname{null}\left(B_{2}\right)$, the previous conditions may be stated in terms of the null spaces of $Z_{1}, Z_{2}, B_{1}$ and $B_{2}$.

In general, of course, it may exist a matrix $A \in \mathbb{C}^{n \times n}$ that maps $Z$ onto $B$ and no solution be possible with $A \in \mathscr{C}$ or $A \in \mathscr{C}^{(a)}$. In particular, if $Z$ is in $\mathcal{C}_{\mathcal{J}}^{2 m \times k}$ and is full rank, that is, $Z_{1}=Z$ and $Z_{2}=O$, then no $A_{c} \in \mathscr{C}$ can map $Z$ onto $B_{2} \neq O$ and no $A_{s} \in \mathscr{C}^{(a)}$ can map $Z$ onto $B_{1} \neq O$. In this case, again from (4.6) and (4.12), we have

$$
\min _{A \in \mathscr{C}}\|A Z-B\|=\sqrt{2}\left\|\widehat{B}_{2}\right\|=\left\|B_{2}\right\|
$$

and

$$
\min _{A \in \mathscr{C}(a)}\|A Z-B\|=\sqrt{2}\left\|\widehat{B}_{1}\right\|=\left\|B_{1}\right\|
$$

Analogously, if $Z$ is full rank and is in $\mathcal{S}_{\mathcal{J}}^{2 m \times k}$, that is, $Z_{1}=O$ and $Z_{2}=Z$, then, from (4.12) we have

$$
\min _{A \in \mathscr{C}}\|A Z-B\|=\left\|B_{1}\right\| \quad \text { and } \quad \min _{A \in \mathscr{C}(a)}\|A Z-B\|=\left\|B_{2}\right\|
$$

Obviously, it is always the case that $\operatorname{rank}\left(Z_{1}\right) \leq \operatorname{rank}(Z)$ and $\operatorname{rank}\left(Z_{2}\right) \leq \operatorname{rank}(Z)$. For the particular case of these ranks being equal, we have 
THEOREM 4.8. If $Z, Z_{1}$ and $Z_{2}$ have the same rank then $\min \|A Z-B\|$ is the same for $A \in \mathscr{C}, A \in \mathscr{C}^{(a)}$ or general $A$.

Proof. From $Z v=0 \Rightarrow Z_{1} v=\frac{1}{2}(Z+i J Z) v=0$ we conclude that $\operatorname{null}(Z) \subseteq \operatorname{null}\left(Z_{1}\right)$; this and the hypothesis $\operatorname{rank}\left(Z_{1}\right)=\operatorname{rank}(Z)$ allows us to state that those null spaces are the same. Similarly, we may say that $\operatorname{null}(Z)=\operatorname{null}\left(Z_{2}\right)$. Therefore, the right singular vectors corresponding to null singular values of $Z, Z_{1}$ (or $\widehat{Z}_{1}$ ) and $Z_{2}$ (or $\widehat{Z}_{2}$ ) are the same. This implies (see Section 5.5) that

$$
\left(I-Z^{\dagger} Z\right)=\left(I-\widehat{Z}_{1}^{\dagger} \widehat{Z}_{1}\right)=\left(I-\widehat{Z}_{2}^{\dagger} \widehat{Z}_{2}\right)
$$

and the residuals expressed in (4.6) and (4.12) are the same. Now, for a general $A$ we write

$$
\begin{aligned}
\left\|B\left(I-Z^{\dagger} Z\right)\right\|^{2} & =\left\|P\left(B_{1}+B_{2}\right)\left(I-Z^{\dagger} Z\right)\right\|^{2} \\
& =2\left\|\left[\begin{array}{c}
\widehat{B}_{2} \\
\widehat{B}_{1}
\end{array}\right]\left(I-Z^{\dagger} Z\right)\right\|^{2} \\
& =2\left(\left\|\widehat{B}_{2}\left(I-Z^{\dagger} Z\right)\right\|^{2}+\left\|\widehat{B}_{1}\left(I-Z^{\dagger} Z\right)\right\|^{2}\right)
\end{aligned}
$$

and, again from (4.19) conclude that the residual is equal to those in (4.6) and (4.12).

We end this section observing that when $Z$ and $B$ are real, the ranks of $Z, Z_{1}$ and $Z_{2}$ are always the same and the previous theorem applies, i.e., there are always real matrices in $\mathscr{C}$ and in $\mathscr{C}^{(a)}$ that attain the same minimum residual as a general matrix. This will be shown in Section 5.3.

5. Algorithms. In this section, we propose algorithms for computing the solutions of Problems I and II, first the case of $A \in \mathscr{C}$ and then the case of $A \in \mathscr{C}^{(a)}$. We also present an algorithm to compute the solutions of Problems I and II for an arbitrary matrix $A \in \mathbb{C}^{2 m \times 2 m}$, the unstructured minimization problem.

5.1. Unstructured minimization. An essential part of the computation of $A$ in (4.2) and the minimum value in (4.1) is the full SVD computation of $Z \in \mathbb{C}^{q \times k}$, with $q \geq k$,

$$
Z=\left[\begin{array}{ll}
U_{1} & U_{2}
\end{array}\right]\left[\begin{array}{ll}
\Sigma & O \\
O & O
\end{array}\right]\left[\begin{array}{ll}
V_{1} & V_{2}
\end{array}\right]^{H}
$$

where, for $\operatorname{rank}(Z)=r<k, U_{1} \in \mathbb{C}^{q \times r}, U_{2} \in \mathbb{C}^{q \times(q-r)}, V_{1} \in \mathbb{C}^{k \times r}, V_{2} \in \mathbb{C}^{k \times(k-r)}$ have orthonormal columns and $\Sigma=\operatorname{diag}\left(\sigma_{1}, \ldots, \sigma_{r}\right)$, with $\sigma_{i}>0, i=1, \ldots, r$, the nonzero singular values of $Z$. When $Z$ is full rank $(r=k),(5.1)$ gives

$$
Z=\left[\begin{array}{ll}
U_{1} & U_{2}
\end{array}\right]\left[\begin{array}{l}
\Sigma \\
O
\end{array}\right] V_{1}^{H}
$$


if $q>k$, and gives place to

$$
Z=U_{1} \Sigma V_{1}^{H},
$$

if $q=k$. Thus, in the full rank case, $V_{2}$ does not exist and, if $p=k, U_{2}$ does not exist either. The following expressions are always valid if we make the convention that in the full rank case $V_{2} V_{2}^{H}$ and $V_{2}^{H} V_{2}$ are null matrices and, if $p=k, U_{2} U_{2}^{H}$ is also a null matrix. This convention will be used throughout Section 5 , including the structured cases.

The Moore-Penrose inverse of $Z$ is

$$
Z^{\dagger}=V_{1} \Sigma^{-1} U_{1}^{H}
$$

We have

$$
\left(I-Z^{\dagger} Z\right)=\left(I-V_{1} \Sigma^{-1} U_{1}^{H} U_{1} \Sigma V_{1}^{H}\right)=\left(I-V_{1} V_{1}^{H}\right)=V_{2} V_{2}^{H}
$$

and

$$
\left(I-Z Z^{\dagger}\right)=\left(I-U_{1} \Sigma V_{1}^{H} V_{1} \Sigma^{-1} U_{1}^{H}\right)=\left(I-U_{1} U_{1}^{H}\right)=U_{2} U_{2}^{H} .
$$

Entering (5.6) in (4.2) we get

$$
A=B V_{1} \Sigma^{-1} U_{1}^{H}+K U_{2} U_{2}^{H}
$$

with $K \in \mathbb{C}^{2 m \times 2 m}$ arbitrary. Using (5.5) in (4.1) we get

$$
\min _{A \in \mathbb{C}^{2 m \times 2 m}}\|A Z-B\|=\left\|B V_{2} V_{2}^{H}\right\| .
$$

Denoting this value by $\sigma$ and defining

$$
\mathcal{S}=\left\{A \in \mathbb{C}^{2 m \times 2 m}:\|A Z-B\|=\sigma\right\},
$$

in Problem II, for a given $\widetilde{A} \in \mathbb{C}^{2 m \times 2 m}$, we want to compute

$$
\min _{A \in \mathcal{S}}\|\widetilde{A}-A\|=\min _{K \in \mathbb{C}^{2 m \times 2 m}}\left\|\widetilde{A}-B V_{1} \Sigma^{-1} U_{1}^{H}-K U_{2} U_{2}^{H}\right\| .
$$

Since $\left(U_{2} U_{2}^{H}\right)^{2}=U_{2} U_{2}^{H}=\left(U_{2} U_{2}^{H}\right)^{H}$, we can apply Lemma 4.3 to conclude that the unique matrix $K$ which gives the minimum norm is

$$
K=\widetilde{A}-B V_{1} \Sigma^{-1} U_{1}^{H} .
$$

Thus, $\min _{A \in \mathcal{S}}\|\widetilde{A}-A\|$ is attained for

$$
A=B V_{1} \Sigma^{-1} U_{1}^{H}+\left(\widetilde{A}-B V_{1} \Sigma^{-1} U_{1}^{H}\right) U_{2} U_{2}^{H}=B V_{1} \Sigma^{-1} U_{1}^{H}+\widetilde{A} U_{2} U_{2}^{H} .
$$

Notice that this is the expression in (5.7) with $K=\widetilde{A}$.

The following algorithm computes the solutions for Problem I (steps 1 and 2) and Problem II (steps 1 and 3 ) for an arbitrary matrix $A \in \mathbb{C}^{2 m \times 2 m}$. 


\section{Algorithm 1}

Given $Z, B, K$ and $\widetilde{A}$, compute:

1. the full SVD of $Z$,

$$
Z=\left[\begin{array}{ll}
U_{1} & U_{2}
\end{array}\right]\left[\begin{array}{ll}
\Sigma & O \\
O & O
\end{array}\right]\left[\begin{array}{ll}
V_{1} & V_{2}
\end{array}\right]^{H}
$$

2. $A=B V_{1} \Sigma^{-1} U_{1}^{H}+K U_{2} U_{2}^{H}$ and the minimum residual $\left\|\left(B V_{2}\right) V_{2}^{H}\right\|$, according to (5.8);

3. $A=B V_{1} \Sigma^{-1} U_{1}^{H}+\widetilde{A} U_{2} U_{2}^{H}$ and the minimum value $\|\widetilde{A}-A\|$.

To give an estimate of the number of complex arithmetic operations involved in the algorithm we will assume that $m$ is significantly larger than $k$. In step 1 the number of flops for the complete SVD is about $16 m^{2} k+22 k^{3}$ and $12 m k^{2}+20 k^{3}$ if $U_{2}$ is not required (using the R-SVD) [4, p.254]. In step 2, the first term in the expression for $A$ requires about $8 m^{2} r$ and the second term is more expensive since it requires about $16 \mathrm{~m}^{3}$ flops. Therefore the solution $A$ of minimal norm for unstructured Problem I, which occurs for the choice $K=O$, is the cheapest to compute, requiring a total of $12 m k^{2}+8 m^{2} r+20 k^{3}$ flops. The computation of the residual costs an extra $8 m k(k-r)$ flops, approximately, which is rather cheap when $r$ is close to $k$. A direct computation of $\|A Z-B\|$ is more expensive (it costs about $8 m^{2} k$ flops) and in our numerical experiments we did not find significant differences in the size of the computed residuals.

The cost of computing $A$ in step 3 is equal to the corresponding cost of step 2 when $K \neq O$. When Problem II is to be solved after Problem I we may reuse the first term in the expression for $A$ in step 2, which is the same in both problems, and also the product $U_{2} U_{2}^{H}$, if it has been computed before. The computation of the minimum value $\|\widetilde{A}-A\|$ costs about $8 m^{2}$ flops.

5.2. Minimization for $A \in \mathscr{C}$ and $A \in \mathscr{C}^{(a)}$. Let $Z, B \in \mathbb{C}^{2 m \times k}$ and $P$ be given in (3.1). Consider $\widehat{Z}_{1}, \widehat{Z}_{2}, \widehat{B}_{1}, \widehat{B}_{2}$ given in (4.5). Let $r_{1}=\operatorname{rank}\left(\widehat{Z}_{1}\right)$ and the full SVD of $\widehat{Z}_{1}$ be

$$
\widehat{Z}_{1}=\left[\begin{array}{ll}
\widehat{U}_{1} & \widehat{U}_{2}
\end{array}\right]\left[\begin{array}{ll}
\widehat{\Sigma} & O \\
O & O
\end{array}\right]\left[\begin{array}{ll}
\widehat{V}_{1} & \widehat{V}_{2}
\end{array}\right]^{H}=\widehat{U}_{1} \widehat{\Sigma} \widehat{V}_{1}^{H},
$$

where $\widehat{U}_{1} \in \mathbb{C}^{m \times r_{1}}, \widehat{U}_{2} \in \mathbb{C}^{m \times\left(m-r_{1}\right)}, \widehat{V}_{1} \in \mathbb{C}^{k \times r_{1}}, \widehat{V}_{2} \in \mathbb{C}^{k \times\left(k-r_{1}\right)}$ have orthonormal columns and $\widehat{\Sigma}=\operatorname{diag}\left(\widehat{\sigma}_{1}, \ldots, \widehat{\sigma}_{r_{1}}\right)$, with $\widehat{\sigma}_{i}>0, i=1, \ldots, r_{1}$, the nonzero singular values of $\widehat{Z}_{1}$. Analogously, let $r_{2}=\operatorname{rank}\left(\widehat{Z}_{2}\right)$ and the SVD of $\widehat{Z}_{2}$ be

$$
\widehat{Z}_{2}=\left[\begin{array}{ll}
\widetilde{U}_{1} & \widetilde{U}_{2}
\end{array}\right]\left[\begin{array}{cc}
\widetilde{\Sigma} & O \\
O & O
\end{array}\right]\left[\begin{array}{ll}
\widetilde{V}_{1} & \widetilde{V}_{2}
\end{array}\right]^{H}=\widetilde{U}_{1} \widetilde{\Sigma}_{V_{1}^{H}}
$$


where $\widetilde{U}_{1} \in \mathbb{C}^{m \times r_{2}}, \widetilde{U}_{2} \in \mathbb{C}^{m \times\left(m-r_{2}\right)}, \widetilde{V}_{1} \in \mathbb{C}^{k \times r_{2}}, \widetilde{V}_{2} \in \mathbb{C}^{k \times\left(k-r_{2}\right)}$ have orthonormal columns and $\widetilde{\Sigma}=\operatorname{diag}\left(\widetilde{\sigma}_{1}, \ldots, \widetilde{\sigma}_{r}\right)$, with $\widetilde{\sigma}_{i}>0, i=1, \ldots, r_{2}$, the nonzero singular values of $\widehat{Z}_{2}$. Then, the Moore-Penrose inverses of $\widehat{Z}_{1}$ and $\widehat{Z}_{2}$ are

$$
\widehat{Z}_{1}^{\dagger}=\widehat{V}_{1} \widehat{\Sigma}^{-1} \widehat{U}_{1}^{H} \quad \text { and } \quad \widehat{Z}_{2}^{\dagger}=\widetilde{V}_{1} \widetilde{\Sigma}^{-1} \widetilde{U}_{1}^{H} .
$$

If both $\widehat{Z}_{1}^{\dagger}$ and $\widehat{Z}_{2}^{\dagger}$ are rank-deficient $\left(r_{1}, r_{2}<k\right)$, we have

$$
\left(I-\widehat{Z}_{1}^{\dagger} \widehat{Z}_{1}\right)=\widehat{V}_{2} \widehat{V}_{2}^{H}, \quad\left(I-\widehat{Z}_{2}^{\dagger} \widehat{Z}_{2}\right)=\widetilde{V}_{2} \widetilde{V}_{2}^{H}
$$

and, according to Theorem 4.4 and (5.11),

$$
\min _{A \in \mathscr{C}}\|A Z-B\|=\sqrt{2}\left(\left\|\widehat{B}_{1} \widehat{V}_{2} \widehat{V}_{2}^{H}\right\|^{2}+\left\|\widehat{B}_{2} \widetilde{V}_{2} \widetilde{V}_{2}^{H}\right\|^{2}\right)^{1 / 2} .
$$

In the full rank case $\left(r_{1}=r_{2}=k\right),\left(I-\widehat{Z}_{1}^{\dagger} \widehat{Z}_{1}\right)=\left(I-\widehat{Z}_{2}^{\dagger} \widehat{Z}_{2}\right)=O$ and we have a null residual. In both cases,

$$
\left(I-\widehat{Z}_{1} \widehat{Z}_{1}^{\dagger}\right)=\widehat{U}_{2} \widehat{U}_{2}^{H}, \quad\left(I-\widehat{Z}_{2} \widehat{Z}_{2}^{\dagger}\right)=\widetilde{U}_{2} \widetilde{U}_{2}^{H}
$$

and, using (4.7) in Theorem 4.4, this minimum is attained with

$$
A=\frac{1}{2}\left[\begin{array}{cc}
M+N & -(M-N) i \\
(M-N) i & M+N
\end{array}\right],
$$

where

$$
M=\widehat{B}_{2} \widetilde{V}_{1} \widetilde{\Sigma}^{-1} \widetilde{U}_{1}^{H}+K_{2} \widetilde{U}_{2} \widetilde{U}_{2}^{H} \quad \text { and } \quad N=\widehat{B}_{1} \widehat{V}_{1} \widehat{\Sigma}^{-1} \widehat{U}_{1}^{H}+K_{1} \widehat{U}_{2} \widehat{U}_{2}^{H},
$$

with $K_{1}, K_{2} \in \mathbb{C}^{m \times m}$ arbitrary.

Now we will consider the computation of the solution for problem II. Consider $\tilde{A} \in$ $\mathbb{C}^{2 m \times 2 m}, \widetilde{A}_{1}$, given in (4.8) and $M_{1}$ and $N_{1}$ given in (4.9). Then, according to Theorem 4.5, $A$ is given by (5.14), where in (5.15) $K_{2}=M_{1}$ and $K_{1}=N_{1}$.

The following algorithm computes the solutions for Problem I (steps 1,2 and 3) and Problem II (steps 1, 2, 4 and 5) for $A \in \mathscr{C}$.

\section{Algorithm 2}

Given $Z, B, K_{1}, K_{2}$ and $\widetilde{A}$, compute:

1. $\widehat{Z}_{1}, \widehat{Z}_{2}, \widehat{B}_{1}$ and $\widehat{B}_{2}$, in (4.5);

2. the full SVD's of $\widehat{Z}_{1}$ and $\widehat{Z}_{2}$, in (5.9) and (5.10);

3. $M$ and $N$ in (5.15), for the given $K_{1}$ and $K_{2}, A$ in (5.14) and the residual with (5.12);

4. $M_{1}$ and $N_{1}$ given in (4.9);

5. $M, N$ and $A$ (as in step 3) for $K_{1}=M_{1}$ and $K_{2}=N_{1}$ and the minimum value $\|\widetilde{A}-A\|$. 
For $A \in \mathscr{C}^{(a)}$, the algorithm is similar to Algorithm 2 and we just point out the differences in the expressions to be used in this case. In step 3 , we use

$$
\begin{aligned}
& M=\widehat{B}_{2} \widehat{V}_{1} \widehat{\Sigma}^{-1} \widehat{U}_{1}^{H}+K_{2} \widehat{U}_{2} \widehat{U}_{2}^{H}, \\
& N=\widehat{B}_{1} \widetilde{V}_{1} \widetilde{\Sigma}^{-1} \widetilde{U}_{1}^{H}+K_{1} \widetilde{U}_{2} \widetilde{U}_{2}^{H}, \\
& A=\frac{1}{2}\left[\begin{array}{cc}
M+N & (M-N) i \\
(M-N) i & -(M+N)
\end{array}\right], \\
& \min _{A \in \mathscr{C}(a)}\|A Z-B\|=\sqrt{2}\left(\left\|\widehat{B}_{2} \widehat{V}_{2} \widehat{V}_{2}^{H}\right\|^{2}+\left\|\widehat{B}_{1} \widetilde{V}_{2} \widetilde{V}_{2}^{H}\right\|^{2}\right)^{1 / 2} .
\end{aligned}
$$

In step 4, use (4.9) for $M_{2}$ and $N_{2}$. In step 5, compute $M, N$ and $A$ as in step 3 for $K_{1}=N_{2}$ and $K_{2}=M_{2}$.

The costs of steps 1 and 4 are relatively inexpensive. In step 2 the number of flops for each one of the two complete SVD's is about $4 m^{2} k+22 k^{3}$ and $6 m k^{2}+20 k^{3}$ if $\widehat{U}_{2}$ and $\widetilde{U}_{2}$ are not required. In step 3 , to compute $M$ and $N$ we need $2 m^{2} r_{1}$ and $2 m^{2} r_{2}$ flops, respectively, if $K_{1}=K_{2}=O$ which, as in the unstructured case, produce the minimal norm solution. Since $r_{1}+r_{2} \leq 2 r$, the total cost to compute $M$ and $N$, in this case, is not larger than $4 m^{2} r$ flops and this is half the cost of computing $A$ in the unstructured case. For general matrices $K_{1}$ and $K_{2}$, the computation of $M$ and $N$ requires in addition $4 m^{3}$ extra flops in contrast to $16 \mathrm{~m}^{3}$ flops in the general case. The explicit construction of $A$ requires $2 m^{2}$ flops. The computation of the residual costs an extra $4 m k\left(k-r_{1}\right)+4 m k\left(k-r_{2}\right)$ flops, approximately. If $r_{1}=r_{2}=r$, this equal the corresponding cost in the unstructured case; however, if $r_{1} \ll r$ or $r_{2} \ll r$, this cost may be larger in the structured case (the extreme situation occurs when $r=k$ and $r_{1}$ or $r_{2}$ are equal to zero).

The cost of computing $M, N$ and $A$ in step 5 equals the corresponding cost in step 3 when $K_{1}, K_{2} \neq O$. As in the unstructured case, when Problem II is to be solved after Problem I, we may reuse the first terms in the expressions for $M$ and $N$ in step 3, which are the same in both problems, and also the products $\widehat{U}_{2} \widehat{U}_{2}^{H}$ and $\widetilde{U}_{2} \widetilde{U}_{2}^{H}$, if computed before. The computation of the minimum value $\|\widetilde{A}-A\|$ costs about $8 m^{2}$ flops.

In conclusion, to produce structured solutions for Problems I and II, our algorithm requires less arithmetic than the algorithm for the general case. As noted before, to compute the minimal norm solution for Problem I, our algorithm is about half as expensive as the general algorithm. For solutions with general matrices $K, K_{1}$ and $K_{2}$ (which is always the case in Problem II), our algorithm requires approximately $1 / 4$ of the number of flops of the general algorithm.

5.3. The real case. Although Algorithm 1 and Algorithm 2 have been presented for complex matrices, there are situations where one may be interested in producing a real solution $A$ (we noted before that real orthogonal sympletic matrices belong to $\mathscr{C}$ ). When $Z$ and $B$ 
are real, Algorithm 1 produces necessarily real matrices $A$ since all the arithmetic involved is real. This is not the case in Algorithm 2. From (5.14) it follows that $A$ is real if and only if $M$ and $N$ in (5.15) are complex conjugate. This happens whenever $Z$ and $B$ are real and we take $K_{1}$ and $K_{2}$ in (5.15) with $K_{1}=\bar{K}_{2}$. Because $\left(Z_{1}, Z_{2}\right)$ and $\left(B_{1}, B_{2}\right)$, in (4.3) and (4.4), respectively, are pairs of conjugate matrices, the same is true for $\widehat{Z}_{1}$ and $\widehat{Z}_{2}$ and the corresponding factors in their decompositions in (5.9) and (5.10), respectively. As a consequence of this, Algorithm 2 gets the following simplifications when $Z$ and $B$ are real: in step 2, one single complex SVD (either of $\widehat{Z}_{1}$ or $\widehat{Z}_{2}$ ) is required and in step 3 only one of $M$ and $N$ is computed. Furthermore, in Problem II, for a real $\widetilde{A}, M_{1}$ and $N_{1}$ in step 4 are also conjugate (see Lemma 3.1). The amount of computation is also reduced in step 5 similarly to step 3.

6. Numerical examples. We implemented our algorithms in MatlaB 7.5.0342 (R2007b), with rounding error unit equal to $2^{-53} \approx 1.1 \times 10^{-16}$. For Problem I, we computed the minimum norm solutions with null matrices $K, K 1$ and $K 2$. In Table 6.1 we summarize the numerical results obtained with our codes for 15 given pairs $(Z, B)$. Here, as before, $A_{c} \in \mathscr{C}, A_{s} \in \mathscr{C}^{(a)}$ and $A$ is a general matrix. The residuals $\mathcal{O}\left(10^{-12}\right), \mathcal{O}\left(10^{-13}\right)$ and $\mathcal{O}\left(10^{-14}\right)$ are due to rounding errors since they correspond to cases for which null residuals are expected, in accordance with Theorem 4.2 and our analysis in section 4.4.

TABLE 6.1

Minimal residuals for problem I

\begin{tabular}{cccccccc} 
& $m, k$ & $\|A Z-B\|$ & $\left\|A_{c} Z-B\right\|$ & $\left\|A_{s} Z-B\right\|$ & $\|A\|$ & $\left\|A_{c}\right\|$ & $\left\|A_{s}\right\|$ \\
\hline \hline 1 & 10,10 & $4.9 \times 10^{-14}$ & $3.3 \times 10^{-14}$ & $3.2 \times 10^{-14}$ & 4.37 & 19.02 & 19.96 \\
2 & 100,20 & $6.7 \times 10^{-14}$ & $1.1 \times 10^{-13}$ & $1.2 \times 10^{-13}$ & 4.73 & 7.09 & 7.06 \\
3 & 1000,200 & $1.0 \times 10^{-12}$ & $1.2 \times 10^{-12}$ & $1.2 \times 10^{-12}$ & 18.26 & 44.57 & 45.00 \\
\hline \hline 4 & 10,10 & $6.8 \times 10^{-14}$ & 7.88 & 8.39 & 11.45 & 8.34 & 7.84 \\
5 & 100,20 & $5.7 \times 10^{-14}$ & 36.39 & 36.40 & 7.01 & 4.93 & 4.99 \\
6 & 1000,200 & $1.6 \times 10^{-12}$ & 365.42 & 365.14 & 22.38 & 15.82 & 15.83 \\
7 & 100,10 & $1.6 \times 10^{-14}$ & $1.4 \times 10^{-14}$ & 26.14 & 2.47 & 2.47 & 0 \\
\hline \hline 8 & 10,10 & 3.80 & 3.80 & 3.80 & 3.56 & 8.45 & 8.76 \\
9 & 50,10 & 8.31 & 18.82 & 18.83 & 4.76 & 3.38 & 3.35 \\
10 & 100,20 & 11.76 & 11.76 & 11.76 & 4.60 & 6.85 & 6.79 \\
11 & 1000,200 & 36.93 & 366.41 & 365.73 & 22.32 & 15.77 & 15.80 \\
\hline \hline 12 & 20,20 & 3.35 & 3.35 & 3.35 & 4.95 & 13.12 & 34.13 \\
13 & 50,10 & $1.4 \times 10^{-14}$ & 18.03 & 17.96 & 3.76 & 2.64 & 2.68 \\
14 & 100,20 & $6.4 \times 10^{-14}$ & $2.3 \times 10^{-13}$ & $2.5 \times 10^{-13}$ & 4.13 & 6.16 & 6.10 \\
15 & 100,20 & $2.1 \times 10^{-13}$ & 32.55 & 32.67 & 6.08 & 4.22 & 4.38 \\
\hline \hline
\end{tabular}

In the first 3 cases, matrices $Z$ and $B$ are generated with Matlab function rand. The 
produced matrix $Z$, as well as the projections $Z_{1}$ and $Z_{2}$ are full rank and consistency is always expected.

In cases $4-7, Z$ has full rank and is either in the subspace $\mathcal{C}_{\mathcal{J}}^{2 m \times k}\left(Z_{2}=O\right)$ or in the subspace $\mathcal{S}_{\mathcal{J}}^{2 m \times k}\left(Z_{1}=O\right)$. Here, matrices $Z$ were generated according to (4.5) from random $(m \times k)$ matrices $\widehat{Z}_{1}$ and $\widehat{Z}_{2}$. The computed residuals are in accordance with (4.16) - (4.18). In particular, in case 7 we used $Z_{2}=B_{2}=O$ and Algorithm 1 produced the same $A_{c} \in \mathscr{C}$ as Algorithm 2. This is because the general solution with minimum norm in this case is necessarily in $\mathscr{C}$. With $A=\widetilde{A}_{c}+\widetilde{A}_{s}, \widetilde{A}_{c} \in \mathscr{C}$ and $\widetilde{A}_{s} \in \mathscr{C}^{(a)}$, we have

$$
\begin{aligned}
\|A Z-B\|^{2} & =\left\|\left(\widetilde{A}_{c}+\widetilde{A}_{s}\right)\left(Z_{1}+Z_{2}\right)-\left(B_{1}+B_{2}\right)\right\|^{2} \\
& =\left\|\widetilde{A}_{c} Z_{1}+\widetilde{A}_{s} Z_{2}-B_{1}\right\|^{2}+\left\|\widetilde{A}_{c} Z_{2}+\widetilde{A}_{s} Z_{1}-B_{2}\right\|^{2} \\
& =\left\|\widetilde{A}_{c} Z_{1}-B_{1}\right\|^{2}+\left\|\widetilde{A}_{s} Z_{1}\right\|^{2}
\end{aligned}
$$

and the minimum residual is attained with $\widetilde{A}_{s}=O$ and $A=\widetilde{A}_{c}$ that minimizes $\left\|\widetilde{A}_{c} Z_{1}-B_{1}\right\|$. That $A_{s}=O$ also minimizes $\left\|A_{s} Z-B\right\|^{2}$ in this case $\left(Z_{2}=B_{2}=O\right)$ is a consequence of its expression in Theorem 4.6.

Cases 8 to 11 are examples of $Z$ being rank-deficient (by making copies of random columns) and $B$ full rank. The problem is never consistent since $A Z$ can not be full rank.

In cases 12 to $15, Z$ and $B$ are both rank deficient and the condition $\operatorname{null}(Z) \subseteq \operatorname{null}(B)$ in Theorem 4.2 only fails in case 12 . In case 14 , we also have $\operatorname{null}\left(Z_{1}\right) \subseteq \operatorname{null}\left(B_{1}\right)$ and $\operatorname{null}\left(Z_{2}\right) \subseteq \operatorname{null}\left(B_{2}\right)$. We adopted a simple method to generate rank deficient consistent problems. For instance, in case 13 the first 6 independent columns of $Z$ and $B$ have been generated randomly and then, for $i=7, \ldots, 10$, the ith column of $Z$ and $B$ is computed using the same linear combination of the first six columns of $Z$ and $B$, respectively.

In cases $8,10,12$ and 14, the ranks of $Z, Z_{1}$ and $Z_{2}$ are the same and, in accordance with Theorem 4.8, we have equal residuals with $A, A_{c}$ and $A_{s}$.

The next table shows results for Problem II with matrix $\widetilde{A}$ generated randomly.

When the smallest residual $\|A Z-B\|$ for unstructured $A$ is also attained for structured $A_{c}\left(A_{s}\right)$ it is obvious that $\|\tilde{A}-A\| \leq\left\|\widetilde{A}-A_{c}\right\|$ and $\|\tilde{A}-A\| \leq\left\|\widetilde{A}-A_{s}\right\|$ because the set of solutions $A_{c}\left(A_{s}\right)$ is contained in the set of general solutions $A$. In case 2 the problem is consistent for A but not for $A_{c}$ or $A_{s}$. In case $3, \widetilde{A} \in \mathscr{C}$ but does not belong to the set of solutions $A_{c}$ which give minimum residual $\left\|A_{c} Z-B\right\|$ (otherwise, $\left\|\widetilde{A}-A_{c}\right\|$ would be zero). In case $5, \widetilde{A} \in \mathscr{C}^{(a)}$ and a similar observation can be made. 
TABLE 6.2

Minimal residuals for problem II

\begin{tabular}{ccccc} 
& $m, k$ & $\|\widetilde{A}-A\|$ & $\left\|\widetilde{A}-A_{c}\right\|$ & $\left\|\widetilde{A}-A_{s}\right\|$ \\
\hline \hline 1 & 10,10 & 15.47 & 21.97 & 21.62 \\
2 & 50,50 & 92.89 & 86.68 & 88.11 \\
3 & 100,10 & 71.91 & 98.90 & 115.25 \\
4 & 100,20 & 141.96 & 153.67 & 153.66 \\
5 & 1000,200 & 758.97 & 1201.40 & 1001.82 \\
\hline \hline
\end{tabular}

7. Conclusions. The problems addressed have been studied before by other authors for different types of structured matrices. We have extended the previous work to other structures which are exhibited by certain matrices that occur in applications. More precisely, for given complex matrices $Z$ and $B$, we proposed algorithms to compute $A \in \mathscr{C}$ (centralizer of $\mathcal{J}$ ) or $A \in \mathscr{C}^{(a)}$ (anti-centralizer of $\mathcal{J}$ ) that minimize $\|A Z-B\|$. We proved several new theoretical results and illustrated them with numerical examples. Also, for such solutions $A$ and for a given matrix $\tilde{A}$, we have considered the problem of minimizing $\|\tilde{A}-A\|$. The key idea of our work has been the use of special orthogonal subspaces that allow the decomposition of each one the minimization problems into two independent subproblems. As a consequence of this, our algorithms not only deliver solutions which are of the required type but they need less arithmetic than previous algorithms for unstructured matrices.

Acknowledgements. We are grateful to Prof. Beresford Parlett from the University of California at Berkeley for reading the manuscript and making several observations that improved our paper significantly.

\section{REFERENCES}

[1] J. R. Bunch. The Weak and Strong Stability of Algorithms in Numerical Linear Algebra. Linear Algebra and its Applications, 88: 49-60, 1987.

[2] A. Bunse-Gerstner, R. Byers and V. Mehrmann. A chart of numerical methods for structured eigenvalue problems. SIAM J. Matrix Anal. Appl. 13: 419-453, 1992.

[3] J. W. Demmel. Applied Numerical Linear Algebra. SIAM, Philadelphia, PA USA, 1997.

[4] G. H. Golub, C. F. Van Loan. Matrix Computations. Johns Hopkins University Press, Baltimore and London, 3rd ed., 1996.

[5] Alan J. Laub. Matrix Analysis for Scientists and Engineers. Society for Industrial and Applied Mathematics, Philadelphia, PA, 2005.

[6] Z. Y. Liu, H. Fassbender. An inverse eigenvalue problem and an associated approximation problem for generalized K-centrohermitian matrices. J. Computational Applied Math., 206(1): 578-585, 2007.

[7] Z. Liu, Z. Tian, Y. Tan. Computing the Least-Square Solutions for Centrohermitian Matrix Problems. Applied Math. Computation, 174:566-577, 2006.

[8] D. S. Mackey, N. Mackey, F. Tisseur. Structured tools for structured matrices. Electr. J. Linear Algebra, 10: $106-145,2003$.

[9] D. S. Mackey, N. Mackey, F. Tisseur. Structured factorizations in scalar product spaces. SIAM J. Matrix 
Anal. Appl., 27:821-850, 2006.

[10] F. Tisseur. A chart of backward errors for singly and doubly structured eigenvalue problems. SIAM J. Matrix Anal. Appl., 24:877-897, 2003.

[11] W. F. Trench. Hermitian, hermitian R-symmetric, and hermitian R-skew symmetric procrustes problems. Linear Algebra and its Applications, 387:83-98, 2004.

[12] W. F. Trench. Minimization problems for (R, S)-symmetric matrices and (R, S)-skew symmetric matrices. Linear Algebra and its Applications, 389:23-31, 2004.

[13] F. Zhou, L. Zhang, Z. Hu. Least-square solution for inverse problems of centrosymmetric matrices. Computers Math. Appl., 45:1581-1589, 2003. 